\title{
Aus dem Vorwort zur 9. Auflage
}

Unser Kommentar wird von wissenschaftlich denkenden Praktikern und praxiserfahrenen Wissenschaftlern geschrieben. Er bemüht sich stets um eine Dogmatik - ein aus der Autorität des EStG entwickeltes System -, eine verlässliche Information über die Gründe, die Wirkungen, auch die Unzulänglichkeiten einer Norm, will aber vor allem eine Verstehenshilfe für das Gesetz, die Rechtsprechung und die Verwaltungsvorschriften sein. Jeder, der das EStG in seinen Planungen und Gestaltungen zu beachten, einen Einspruch, eine Klage oder Revision zu begründen, der Finanzverwaltung ein kritischer Gesprächspartner zu sein, seinem Mandanten das Gesetz zu erklären hat oder es in eigener Sache verstehen will, wird - so hoffen wir - in unseren Kommentierungen Wissen, Anregungen, Warnungen und strukturelle Nachdenklichkeiten finden.

Die Einkommensteuer begründet ein Dauerrechtsverhältnis. Sie begleitet uns das gesamte Erwerbsleben im Erfolg von Gewinn oder auch Verlust, braucht deshalb eine nachhaltige Grundlage für den Umgang mit Gesetz und Recht. Wir bemühen uns in unserem Autorenkreis um diese Kontinuität. Die jährliche Auflage unseres Kommentars bildet grundsätzlich den Rechtsstand zum 1. Januar des Erscheinungsjahres ab und berücksichtigt alle erheblichen Gesetzesänderungen und die wesentliche Rechtsprechung seit dem Erscheinen der Vorauflage.

... Nun beginnen wir mit der 9. Auflage beim Verlag Dr. Otto Schmidt KG, Köln, eine grundlegende Erneuerung unseres Kommentars, der in Konzeption, Anspruch und Anliegen identisch bleibt, in der Art der Präsentation, der erleichterten Zugänglichkeit nicht zuletzt durch eine elektronische Version, der Lesbarkeit und Praxisnähe sich aber wesentlich verbessern dürfte. Anregungen, Einwände und Ergänzungen sind uns stets willkommen. Wir freuen uns zusammen mit dem Verlag auf den neuen gemeinsamen Weg.

Heidelberg, im Februar 2010

Paul Kirchhof 